Methods In 2016, a regional group of experts in sanitary alerts in occupational health was involved as well as experts in air treatment, indoor air quality, building materials, and occupational medicine. Investigations were structured into 4 axes : clinical, epidemiological, environmental and psychosociological, all accepted by a local steering committee, except the psycho-sociological investigations.

Results Out of 130 workers of the ECF, 24 met the case definition. Among them, 18 worked in the newly rehabilitated building. Cases occurred regularly in this building from 2009 to 2016. Symptoms increasing with damp weather were: fatigue (89\%), headache $(83 \%)$ and multi-site irritations (35 to $88 \%$ ). Medical consultations were reported by 12 workers. Underground springs existed under the building and water ingresses occurred after raining. Molds in high concentrations in indoor air ( $\left.>1000 \mathrm{UFC} / \mathrm{m}^{3}\right)$ were identified in some offices. Ventilation systems followed reglementation. A surface disinfectant was withdrawn due to toxic compounds. Alcans disappeared when flooring was replaced in an office.

Conclusion This episode of sick building syndrom had an exceptional duration. Although former remediation measures were undertaken, the dampness of the environnment and symptoms of workers persisted. This suggests 2 possible ways of symptoms chronicization : hyper-sensibilisation of workers due to identified molds and/or psycho-sociological runaway (unfortunately not investigated).

\section{P-119 OCCUPATIONAL RADIATION-INDUCED CANCER IN MEDICAL STAFF (ORICAMS): PROTOCOL FOR A FRENCH NESTED CASE-CONTROL STUDY}

'Julie Lopes, Clémence Baudin, Juliette Feuardent, Hervé Roy, Sylvaine Caer-Lorho, Klervi Leuraud, Marie-Odile Bernier. 'Insitute of Radiobiological Protection and Nuclear Safety, France

\subsection{6/OEM-2021-EPI.205}

Introduction Currently, medical workers represent the largest group of workers occupationally exposed to ionizing radiation (IR), reaching 7.4 million practitioners worldwide. The risk of radiation associated cancer is still under debate for low-doses IR exposures for these professionals. Some publications have reported an excess of central nervous system (CNS) tumors in interventional cardiologists, but the link with the profession has never been established in a large, properly controlled epidemiological study.

Objectives The ORICAMs (Occupational Radiation-Induced Cancer in Medical Staff) nested case-control study aims to assess the dose-response relationship between IR exposure and CNS tumor.

Methods This study includes medical workers with at least one dosimetric record in the national dose registry called SISERI (Système d'Information de la Surveillance de l'Exposition aux Rayonnements Ionisants) between 2002 and 2012. Follow-up ended in 2019. Individual cumulative doses for each worker will be provided by SISERI. CNS death cases will be matched 1:5 by gender, year of birth, and date of enrollment, with controls alive at the time of case death. Associations between CNS cancer death and cumulative dose will be investigated using conditional logistic regressions.
Results The study will quantify the relationship between occupational exposure to low doses of IR and CNS tumor mortality risks in medical workers. The inclusion of 45 CNS cases and their matched controls will allow detecting at least an odds ratio of 1.5 for a statistical power of $80 \%$ and an alpha significance level of $5 \%$.

Conclusion This study will improve the knowledge of the health risks associated with repeated low-dose IR exposures and will contribute to improve radiation protection strategies for medical workers. This cohort will be part of the international BECOME (Brain cancEr risk in pooled Case-cOntrol study of MEdical workers) project, which will address the same objectives, but using joint analyses of data from France, South Korea and the United States.

\section{P-126 ASSESSMENT OF MORAL HARASSMENT AT WORKPLACE IN TUNISIAN JUNIOR DOCTORS THROUGH A TWO-STEP STUDY}

'Samia Machghoul, Marouen Hayouni, Ines Rassas, Feten Mhenni, Nidhal Mlik, Imen Miled Charfeddine Amri, Neila Chaari, Adnene Hanchi, Mohamed Akrout, Irtyah Merchaoui. ${ }^{1}$ Faculty of Medicine of Monastir, Tunisia

\subsection{6/OEM-2021-EPI.206}

Introduction The Tunisian revolution was followed by a flourishing in the freedom of expression, which sometimes exceeds the limits. This resulted in an increase of violence levels, particularly in hospitals, worsening already unfavorable work conditions and exposing young doctors to mental harassment at work (MHW).

Objectives To compare the prevalence and the perception of workplace bullying in interns and residents before and after the revolution and to determine its influence on their quality of life.

Methods A two-step-cross-sectional study, carried out in 2009 and in 2016, among 547 and 667 interns and residents respectively. Data concerning sociodemographic and occupational information were collected using a self-administered questionnaire. Workplace bullying was assessed using the Negative Acts Questionnaire Revised (NAQ-R). A person is considered a victim of psychological harassment if he or she has been suffering of any of these acts at least once per week in the last six months. The impact of MHW on the interns and residents' quality of life was determined using the SF8 'health survey' scale.

Results The prevalence of workplace bullying decreased significantly between 2009 (74\%) and 2016 (43.6\%) (p <10 $\left.0^{-3}\right)$. It was related to the professional status, gender, seniority, deliberate choice of medicine, satisfaction, serious family problems and hobbies in 2009 while it was related to professional status, nature of specialty, deliberate choice of medical studies and the satisfaction of the practice of Medicine in 2016. Median mental and physical quality of life scores were below the mean baseline scores in both cohorts with no significant difference; and were both significantly influenced by workplace bullying in the two cohorts.

Conclusion Despite the decrease of workplace bullying among young doctors after the Tunisian revolution, its deleterious impact remains troublesome. The promulgation of incriminating legislation and raising awareness about MHW are urgent preventive measures. 